\title{
Aspectos clínicos e histológicos para o diagnóstico do cisto ósseo simples: relato de caso
}

Clinical and histological aspects for the diagnosis of simple bone cyst: case report

Aspectos clínicos e histológicos para el diagnóstico de quiste ósseo simple: reporte de caso

Ítalo de Lima FARIAS ${ }^{1}$

Elma Mariana Verçosa de MELO SILVA ${ }^{1}$

Tiburtino José de LIMA NETO ${ }^{1}$

Paulo Rogério Ferreti BONAN ${ }^{2}$

Sirius Dan INAOKA ${ }^{3}$

Davi Felipe Neves COSTA ${ }^{3}$

${ }_{1}^{1}$ Residente de Cirurgia e Traumatologia Buco-Maxilo-Facial, Hospital Universitário Lauro Wanderley (UFPB) 58051-900 João Pessoa - PB, Brasil

${ }^{2}$ Docente do Departamento de Odontologia Clínica e Social (DOCS) da Universidade Federal da Paraíba (UFPB), 58051-900 João Pessoa - PB, Brasil ${ }^{3}$ Cirurgião Bucomaxilofacial Hospital Universitário Lauro Wanderley (HULW) 58051-900 João Pessoa - PB, Brasil

\section{Resumo}

Introdução: O cisto ósseo simples é uma lesão benigna intra-óssea considerada um pseudocisto, de etiologia incerta, crescimento lento, radiograficamente radiolúcida e de tratamento simples. Porém, pode fazer diagnóstico diferencial com outras lesões mais agressivas, e a ausência ou tênue presença de uma cápsula cística prejudica seu diagnóstico histopatológico. Objetivo: discutir os detalhes dos achados clínicos, radiográficos, histopatológicos e cirúrgicos para o cisto ósseo simples, bem como, o acompanhamento e o desfecho de um caso em corpo mandibular. Material e Método: Estudo descritivo de relato de caso. Resultados: foi realizada exploração cirúrgica e ostectomia periférica em lesão mandibular radiolúcida, observando-se no trans-cirúrgico discreta presença de material intralesional enviado para análise histopatológica. Conclusão: apesar do tratamento ser simples, o diagnóstico do cisto ósseo simples continua sendo um desafio, onde a exploração cirúrgica é o método mais preciso de diagnóstico e tratamento, mas a avaliação histopatológica é indispensável para exclusão de outras patologias.

Descritores: Cistos Ósseos; Mandíbula; Diagnóstico.

\section{Abstract}

Introduction: Simple bone cyst is a benign intraosseous lesion considered a pseudocyst of uncertain etiology, slow growth, radiographically radiolucent and simple manageable. However, it can make a differential diagnosis with others more aggressive lesions, and the absence or slight presence of a cystic capsule impairs its histopathological diagnosis. Objective: argue about the details of clinical, radiographic, histopathological and surgical findings for simple bone cyst, as well as the follow-up and outcome of a case in mandibular body. Material and Method: Descriptive case report study. Results: It was performed surgical exploration and peripheral ostectomy in a radiolucent mandibular lesion. In transoperative period, a slight presence of intralesional material was observed and sent to histopathological analysis. Conclusion: Although a simple treatment, the diagnosis of simple bone cyst remains a challenge, where surgical exploration is the most accurate method for diagnosis and treatment, but histopathological evaluation is extremely necessary to exclude other pathologies.

Descriptors: Bone Cysts; Mandible; Diagnosis.

\section{Resumen}

Introducción: El quiste óseo simple es una lesión intraósea benigna que se considera un seudoquiste de etiología incierta, crecimiento lento, radiolúcido radiológicamente y tratamiento simple. Sin embargo, puede hacer un diagnóstico diferencial con otras lesiones más agresivas, y la ausencia o leve presencia de una cápsula quística deteriora su diagnóstico histopatológico. Objetivo: Discutir los detalles de los hallazgos clínicos, radiografias, histopatológicos y quirúrgicos para quiste óseo simple, como el seguimiento y el resultado de un caso en el cuerpo mandibular. Material Y Método: estudio descriptivo del informe del caso. Resultados: fue realizada exploración quirúrgica y ostectomía periférica en una lesión mandibular radiolúcida. Fue observado durante el período transoperatorio una ligera presencia de material intralesional enviado para análisis histopatológico. Conclusión: Aunque el tratamiento es simple, el diagnóstico de quiste óseo simple sigue siendo un desafío, donde la exploración quirúrgica es el método más preciso de diagnóstico y tratamiento, pero la evaluación histopatológica es indispensable para excluir otras patologías.

Descriptores: Quistes Óseos; Mandíbula; Diagnóstico.

\section{INTRODUÇÃO}

A classificação da Organização Mundial da Saúde $\quad(\mathrm{WHO})^{1}$ de tumores odontogênicos categorizou o Cisto Ósseo Simples (COS) como uma lesão óssea não neoplásica ${ }^{2}$. Esta foi inicialmente descrita como entidade em 1926 por Lucas e Blum, sendo seus critérios diagnósticos estabelecidos apenas em 1946 por Rushton ${ }^{3}$.

Esse cisto apresenta como sinônimos: cisto ósseo solitário, cisto ósseo traumático, cisto hemorrágico, cisto ósseo hemorrágico, cisto ósseo unicameral e cavidade óssea idiopática ${ }^{1}$. É relativamente incomum, com uma prevalência relatada de $0,6 \%$ (de um estudo de 3353 cistos ósseos) ${ }^{4}$, ocorrendo com mais frequência em homens, apresentando-se entre segunda e terceira décadas ${ }^{5,6}$. Esta lesão ocorre prevalentemente em ossos longos como a porção proximal do úmero e fêmur. Porém, pode também ocorrer no esqueleto craniofacial com prevalência menor que $2 \%$. Quando presente, acomete mais a região de corpo mandibular ${ }^{8}$.
É classicamente descrita como lesão assintomática, de crescimento lento, radiolúcida unilocular bem definida, sem causar expansão das corticais ósseas e sem reabsorção dos dentes adjacentes ou envolvidos na lesão. ${ }^{3,9}$ Porém, pode aparecer como lesão multilocular, associada a dentes impactados, podendo causar expansão das corticais ósseas e assimetria facial ${ }^{10}$.

Sendo definido como um cisto intraósseo, que apresenta uma cápsula tênue ou um tecido conectivo sem epitélio, possui etiologia e patogênese incerta. O termo "cisto" é geralmente mal aplicado, visto que grande parte das lesões são cavidades vazias, contendo não mais que um pequeno fluido seroso ou serosanguinolento, estando o revestimento epitelial ausente $e^{6,11}$.

Hemorragia intramedular pós-traumática é uma das principais hipóteses do gênesis desta, seguida por anormalidade no crescimento ósseo, bloqueio na drenagem venosa ou linfática $\mathrm{e}$ 
degeneração tumoral ${ }^{6}$. A exploração cirúrgica de lesões suspeitas é uma ferramenta determinante para o seu diagnóstico e exclusão de outras patologias com características semelhantes, bem como para quando confirmado o COS, possibilitar o tratamento por meio de curetagem. O acompanhamento radiográfico é também importante para analisar o curso de resolução destas lesões ${ }^{6,9,10}$.

De acordo com os estudos de Chrcanovic e Gomez $^{12}$ em revisão sistemática, recidivas destas lesões estão mais associadas a procedimentos cirúrgicos que não permitem o completo acesso a lesão, como em cavidades grandes que não formam coágulos sanguíneos suficientes para neoformação óssea, na presença de escalopes e em múltiplos cistos.

Assim, o objetivo deste estudo é relatar os detalhes dos achados clínicos, radiográficos, histopatológicos e cirúrgicos, bem como, o acompanhamento e o desfecho de um Cisto Ósseo Simples na mandíbula, enfatizando a importância do conhecimento desta lesão para o diagnóstico diferencial com outras entidades patológicas que mimetizam as mesmas características clínicas e radiográficas.

\section{CASO CLÍNICO}

Paciente do sexo masculino, 33 anos de idade, foi encaminhado de um atendimento odontológico privado ao serviço de Cirurgia BucoMaxilo-Facial do Hospital Universitário Lauro Wanderley da Universidade Federal da Paraíba, devido a uma imagem radiolúcida encontrada em um exame radiográfico de rotina. O paciente não possuía histórico de trauma local ou patologias antecedentes, e nem queixas de sintomatologia dolorosa ou tempo de evolução desta lesão. Ao exame físico, não apresentava abaulamento das corticais ósseas mandibulares ou alterações teciduais (Figura 1).

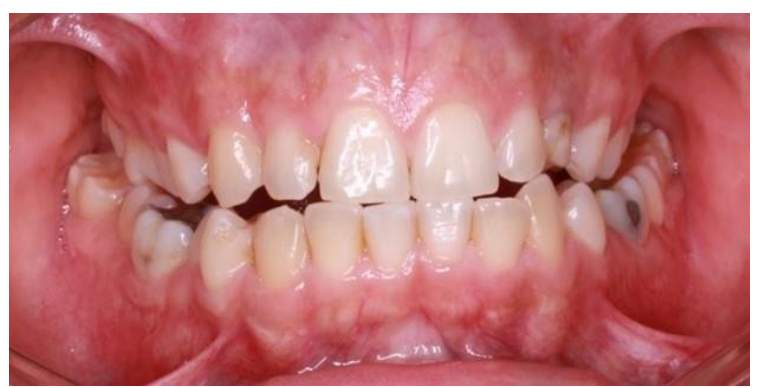

Figura 1: Aspecto clínico mostrando tecidos orais sem alterações das corticais ósseas.

$\mathrm{Na}$ radiografia panorâmica dos maxilares (Figura 2) foi observada imagem radiolúcida, com halo esclerótico, aspecto multilocular, semelhante a "bolhas de sabão", estendendo-se da região distal do elemento dentário 48 ao ramo mandibular direito, sem sinais de reabsorção radicular ou deslocamento dentário, com discreta expansão da cortical óssea alveolar, distal ao 48, e laudo radiográfico com hipótese diagnóstica de ameloblastoma.

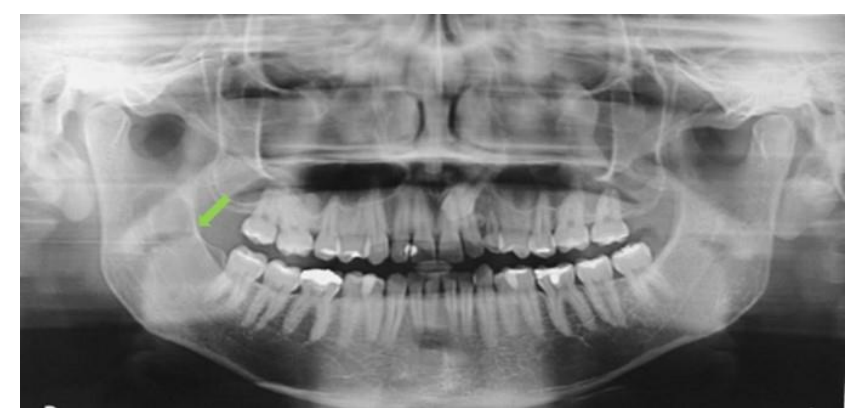

Figura 2: Radiografia panorâmica dos maxilares evidenciando lesão radiolúcida multilocular posterior ao dente 48 em região de ramo mandibular direito; a seta verde evidencia uma região com pequena expansão óssea.

$\mathrm{O}$ teste de vitalidade no dente adjacente a lesão foi positivo, e este não apresentou mobilidade. Foi realizada uma punção com uma fina agulha, porém, nenhum material foi aspirado. Após essas informações, seguiu-se com a exploração cirúrgica, rebatendo o retalho mucoperiosteal, e, através da osteotomia, foi removido parte do teto ósseo da loja cística para biópsia incisional. Em seguida, foi observada uma cavidade vazia sem a presença de tecido fibroso (Figura 3a), prosseguindo então com a curetagem vigorosa de todas as paredes císticas, concluindo-se com a sutura em pontos simples isolados (Figura 3b). A peça cirúrgica foi enviada para análise histopatológica (Figura 4), onde foi observado tecido ósseo lamelar, lacunas de Howship, tecido conjuntivo com fibras colágenas, entremeadas com fibroblastos e vasos sanguíneos, com diagnóstico final de Cisto Ósseo Simples (Figura 5). Após 3 meses da biópsia, observou-se, por meio de acompanhamento radiográfico, redução no tamanho da cavidade cística (Figura 6), e, após 7 meses da cirurgia, foi realizada uma tomografia computadorizada da região para verificar o nível de neoformação óssea, onde foi detectada evidência de regeneração óssea total no seguimento (Figura 7).
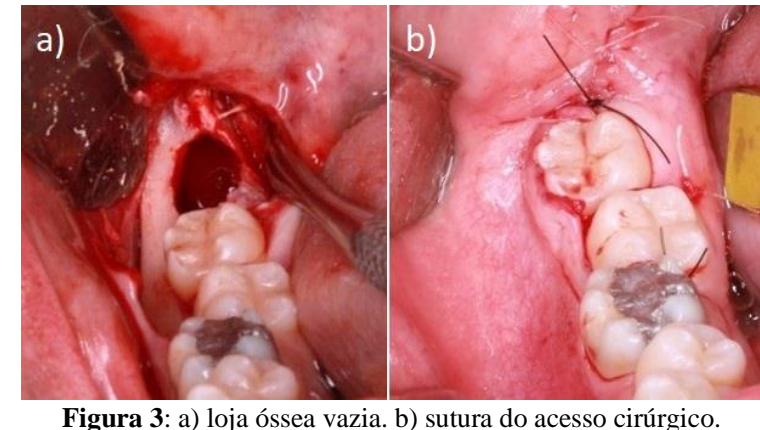

Figura 3: a) loja óssea vazia. b) sutura do acesso cirúrgico.

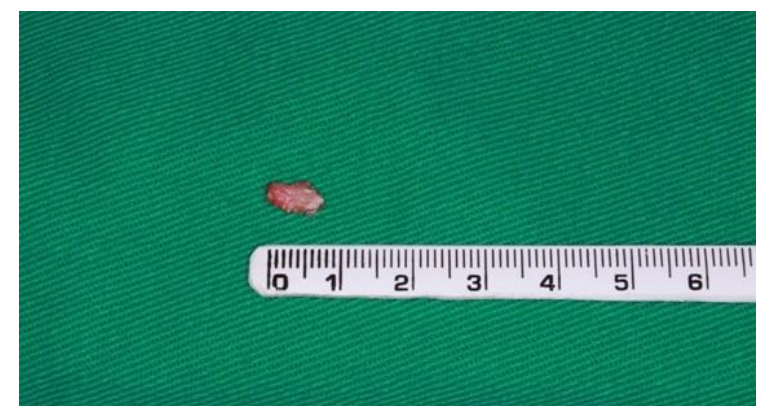

Figura 4: Fragmento ósseo suprajacente a lesão enviado para análise histopatológica. 


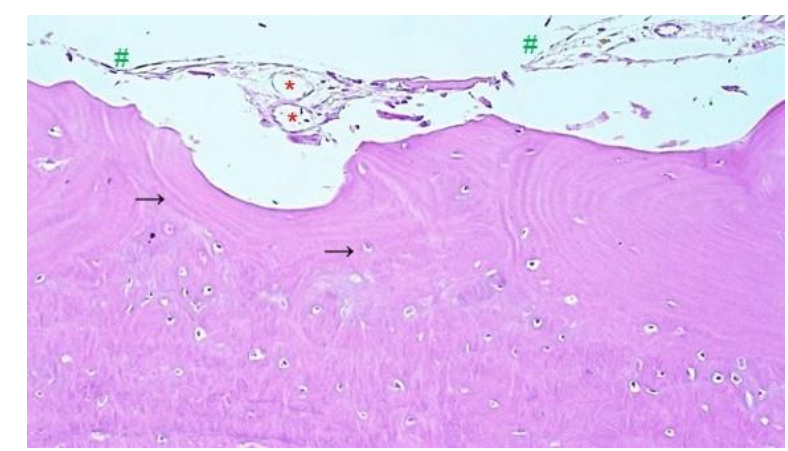

Figura 5: Fotomicrografia da cortical óssea suprajacente a lesão onde observa-se tecido ósseo lamelar com lacunas preenchidas por osteócitos típicos $(\rightarrow)$, tecido conjuntivo fibroso frouxo entremeado por fibroblastos (\#) e vasos sanguíneos (*), cujos achados patológicos são compatíveis com Cisto Ósseo Simples.

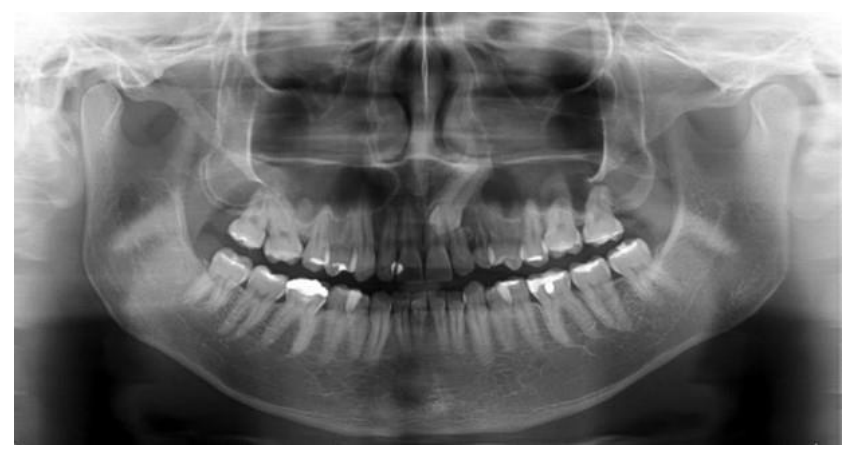

Figura 6: Radiografia panorâmica dos maxilares 3 meses pós-operatório.

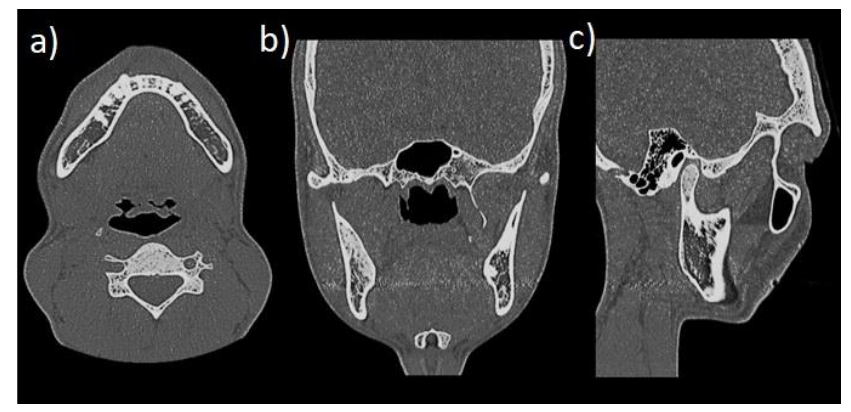

Figura 7: Imagens planares da tomografia computadorizada de face do paciente com 7 meses de pós-operatório, onde observa-se respectivamente nos cortes axial (a), sagital (b) e coronal (c) evidências de neoformação óssea total e remissão da lesão.

\section{DISCUSSÃO}

O cisto ósseo traumático é uma lesão osteolítica, de diagnóstico difícil, caracterizada por crescimento lento e assintomático. Radiograficamente apresenta-se como uma cavidade geódica ou polimorfa, radiolúcida, unilocular, com mínima expansão mandibular, mas que pode apresentar traços radiopacos em pacientes mais velhos com hiperplasia cementária, displasia ou perda da lâmina dura em caso de envolver dentes ${ }^{6,13}$.

A lesão em questão neste relato apresentou características clínicas comuns ao COS, como localização em corpo mandibular e discreto o adelgaçamento da cortical óssea (expansão óssea) e dentes adjacentes a lesão com vitalidade pulpar, ${ }^{6,12-14}$. Porém, radiograficamente apresentou aparência multilocular divergindo da literatura.

Com relação à etiopatogênese da lesão, dentre tantas teorias as principais sugerem associação dos casos relatados na literatura predominantemente com três possíveis origens: anormalidade do crescimento ósseo, degeneração tumoral e traumatismo hemorrágico por fator particular ${ }^{13}$.

Ankha et al. ${ }^{15}$ relataram uma possível associação entre a etiologia traumática do cisto ósseo simples e torcicolo muscular congênito, onde o encurtamento do músculo esternocleidomastoideo durante o parto ou desenvolvimento levou a alterações posturais contribuindo para mudanças de crescimento e desenvolvimento, com desequilíbrio da pressão sobre os ossos do crânio e da face com consequente remodelação e hipoplasia.

Alterações no metabolismo do cálcio associadas a outras alterações do paciente, como algumas síndromes (síndromes dos arcos branquiais, Tetralogia de Fallot) e displasia, foram descritas na literatura com alguma possível relação com o Cisto ósseo simples ${ }^{6,16}$.

Outra teoria sugere alterações vasculares como fator etiopatogênico, uma vez que frequentemente encontra-se na análise microscópica necrose celular asséptica e remodelamento ósseo, o que pode ser ligado à isquemia local por alteração arterial. A alteração da drenagem venosa leva ao acúmulo de líquido intracístico, levando ao aumento da pressão e consequente crescimento cístico. A ausência de conteúdo cístico pode indicar o término da evolução dessa lesão ${ }^{13}$.

A análise citológica de conteúdos sanguinolentos ou serosanguinolentos encontrados nestas lesões como elementos sanguíneos e hemossiderina indicam que em algum momento estas lesões foram preenchidas de sangue. Porém, a gênese da lesão em questão permanece incerta, uma vez que o paciente não apresentava alterações teciduais locais evidentes como remodelação óssea, presença de outras lesões associadas ou histórico de traumas prévios 9 .

Expansão óssea e recidiva são mais frequentes em lesões múltiplas, caracterizando-se mais agressivas. Na primeira década de vida, estas lesões apresentam-se mais agressivas e com maior taxa de recorrência que na adolescência ${ }^{12}$.

O COS faz diagnóstico diferencial com importantes lesões benignas e malignas, como: ameloblastoma, ceratocisto, mixoma, cisto ósseo aneurismático, cisto ósseo solitário, granuloma eosinofílico, granuloma central de células gigantes, tumor marrom do hiperparatireoidismo, osteosarcoma e mieloma múltiplo ${ }^{17}$. Devido as características clínicas e radiográficas, como crescimento lento, assintomático, aspecto multilocular, semelhante a "bolhas de sabão, levantou-se a hipótese diagnóstica de ameloblastoma.

Quando se suspeita da presença de cistos ósseos simples associado a sinais e sintomas brandos, pode-se optar por abordagens mais conservadoras até confirmar-se o diagnóstico ${ }^{17}$. Porém, o caso em 
questão apresentava características clínicas e radiográficas incertas, optando-se pela punção aspirativa, seguida por exploração cirúrgica para confirmação do diagnóstico.

A presença de fluídos no interior da lesão pode ocorrer em até $50 \%$ dos casos $^{12}$. Porém, no presente caso não foi encontrado conteúdo intralesional durante a punção aspirativa por agulha fina. Ao realizar exposição da lesão, também não foi encontrado conteúdo cístico como cápsula cística, o que levantou a hipótese diagnóstica cirúrgica de cisto ósseo simples.

O diagnóstico desta lesão por meio de análise histopatológica é um desafio, pois não possui características histológicas específicas, já que o COS apresenta uma escassa ou delgada cápsula de tecido conectivo fibroso ${ }^{10}$. Tecido inflamatório granulomatoso pode ser encontrado no interior destas lesões, mas as paredes ósseas da lesão não possuem cápsula cística, mas apenas um tecido conectivo fibroso $^{7,18}$.

Rivero et al. ${ }^{10}$ realizaram análise citológica e histológica de cistos ósseos idiopáticos por meio da técnica de coleta de blocos celulares, onde observou a presença de uma rede de fibrina, eritrócitos e células inflamatórias com predominância de linfócitos, macrófagos e neutrófilos. Observou-se ainda a presença de um escasso tecido conectivo, trabeculado ósseo, focos hemorrágicos e hemossiderina. A presença de células gigantes multinucleadas foi escassa e não se observa a presença de uma cápsula epitelial.

No presente caso, como não havia material intralesional para análise histopatológica, foi encaminhado a cortical óssea suprajacente a lesão. Os achados histopatológicos são compatíveis com o diagnóstico de cisto ósseo simples e só foram possíveis devido ao material encaminhado.

A taxa de recorrência desta lesão é em torno de $22 \%$, especialmente em pacientes com displasia cemento-óssea ou lesões múltiplas associadas, sendo recomendado acompanhamento por cerca de 12 a 17 meses, para ser observada neoformação óssea no lugar da lesão, onde a cura é decretada após completa remissão dos sinais de recuperação óssea, o que pode levar até cerca de 3 anos ${ }^{19}$.

Após 7 meses de acompanhamento radiográfico, não ficou evidenciado na tomografia computadorizada sinais de recidiva do COS, cujas características radiográficas poderiam incluir lesão radiolúcida de bordas irregulares com ligeira corticação, podendo ou não ocorrer presença de septos ósseos e deslocamentos dentários ${ }^{20}$.

Outras formas de tratamento que podem ser adicionadas a curetagem em lesões mais extensas é a utilização da engenharia tecidual com enxertos autógenos ou xenógenos para acelerar a reparação óssea ${ }^{10}$.

\section{CONSIDERAÇÕES FINAIS}

A origem dos cistos ósseos simples por traumas ou hemorragias ainda continua sem uma definição clara na literatura. Como as características clínicas e radiográficas desta lesão se assemelham a outras entidades patológicas importantes que podem ter caráter agressivo, a exploração cirúrgica com curetagem ainda é o procedimento de escolha para o seu correto diagnóstico e tratamento, tendo em vista a exclusão de outras doenças, e reduzir ou prevenir danos a estruturas adjacentes que o cisto pode causar de acordo com sua localização e expansão. Além disso, o acompanhamento radiográfico do paciente é importante para assegurar a completa cura, e deve acontecer em até 3 anos, que é o período em que recidivas podem ocorrer.

\section{REFERÊNCIAS}

1. World Health Organization (WHO). Classification of tumours - Pathology and Genetics of Head and Neck Tumours. 3rd edition. Lyon: IARC Press; 2005.

2. Main DM. Epithelial jaw cysts: 10 years of the WHO Classification. J Oral Pathol. 1985;14(1):1-7.

3. Rushton MA. Solitary bone cysts in the mandible. Br Dent J. 1946;81(2):37-49.

4. Seehra J, Horner K, Sloan P. The unusual cyst: solitary bone cyst of the jaws. Dent Update. 2009;36(8):502-8.

5. Chell M, Idle M, Green J. Case report: an unusual finding of a solitary bone cyst in a patient with a fractured mandible. Dent Update. 2015; 42(10):977-78.

6. Wong-Romo G, Carrillo-Terán E, Ángeles-Varela E. Solitary mandibular bone cyst. Case report and literature review. Rev Odont Mex. 2016;20(2):e112-19.

7. Andrade EL, Cerqueira LS, Rebouças DS, Ferreira TG, Marchionni AMT. Cisto ósseo simples: relato de caso. Rev cir traumatol bucomaxilo-fac. 2016;16(2):36-9.

8. Horne RP, Meara DJ, Granite EL. Idiopathic bone cavities of the mandible: an update on recurrence rates and case report. Oral Surg Oral Med Oral Pathol Oral Radiol. 2014;117(2):e71-3.

9. Resnick CM, Dentino KM, Garza R, Padwa BL. A management strategy for idiopathic bone cavities of the jaws. J Oral Maxillofac Surg. 2016;74(6):1153-58.

10. Rivero ERC, Daltoé FP, Mello FW, de Souza CECP, Grando LJ. Aspiration and cytological evaluation of idiopathic bone cavities of the jaw. Tissue Cell. 2017;49(3):435-39.

11. MacDonald-Jankowski DS. Traumatic bone cysts in the jaws of a Hong Kong Chinese population. Clin Radiol.1995;(50):787-91.

12. Chrcanovic BR, Gomez RS. Idiopathic bone cavity of the jaws: an updated analysis of the 
cases reported in the literature. Int $\mathbf{J}$ Oral Maxillofac Surg. 2019;48(7):886-94.

13. Harnet JC, Lombardi T, Klewansky P, Rieger J, Tempe MH, Clavert JM. Solitary bone cyst of the jaws: a review of the etiopathogenic hypotheses. J Oral Maxillofac Surg. 2008; 66(11):2345-48.

14. Silveira HA, Cardoso CL, Pexe M, Araujo RZ, Condezo AB, Curi MM. Simple bone cyst in a 7year-old child. RGO Rev Gauch Odontol. 2017;65(1):83-6.

15. Ankha MEA, Nascimento R, Raldi F, Moraes M, Ribeiro Z, Santos L. Traumatic bone cyst and congenital muscular torticollis: association or a chance? Rev Esp Cir Oral Maxilofac. 2017;39(1):28-49.

16. Ishimoto S, Tanaka S, Maatsishita Y, Kogo M. Mandibular simple bone cyst in a patient with first and second branchial arch syndrome. J Oral Maxillofac Surg Med Pathol. 2017;29(6):538-41.

17. Saia G, Fusetti S, Emanuelli E, Ferronato G, Procopio O. Intraoral endoscopic enucleation of a solitary bone cyst of the mandibular condyle. Int J Oral Maxillofac Surg. 2012;41(3):317-20

18. Bai XF, Hu ZP, Xu Z, Wushou A. Solitary bone cyst of the zygomatic bone. J Oral Maxillofac Surg Med Pathol. 2015;27(2):213-15.

19. Suei Y, Taguchi A, Tanimoto K. Simple bone cyst of the jaws: Evaluation of treatment outcome by review of 132 cases. J Oral Maxillofac Surg. 2007;65(5):918-23.

20. Mathew R, Omami G, Gianoli D, Lurie A. Unusual cone-beam computerized tomography presentation of traumatic (simple) bone cyst: case report and radiographic analysis. Oral Surg Oral Med Oral Pathol Oral Radiol. 2012;113(3):410-13.

\section{CONFLITO DE INTERESSES}

Os autores declaram não haver conflitos de interesse.

\section{AUTOR PARA CORRESPONDÊNCIA}

\section{Ítalo de Lima Farias}

italolimaf@hotmail.com
Submetido em 17/12/2019

Aceito em 18/03/2020 